\title{
Lived Experiences of Patients with COVID-19 in the Kurdistan Region, Iraq: A Qualitative Study
}

\author{
Sarhang Qadir Ibrahim ; Department of Nursing, College of Nursing, Hawler Medical University, Erbil, Iraq. \\ (Correspondence: sarhang.ibrahim@hmu.edu.krd)
}

\section{ABSTRACT}

Background and objective: COVID-19 has adverse effects on physical and mental health and threatens the quality of life significantly; therefore, it is necessary to help COVID-19 patients with their lifestyle, which is tightly bound to the quality of their lives. The present study aims to develop plans to improve the quality of life among COVID-19 patients by analyzing their lived experiences.

Methods and patients: The present qualitative study was conducted from August to September 2020 on twelve COVID-19 patients hospitalized in Erbil's Emirates Hospital, the Kurdistan Region of Iraq. In-depth semi-structured interviews (12) were utilized to collect data. Trustworthiness was ensured by making an excellent prolonged relationship with the patients, peer-checking, member-checking, and implementing experts' suggestion at every stage. Four main themes were extracted from the interviews, which were transcribed verbatim and analyzed using the six methodological activities proposed by Van Manen.

Results: The present study results indicated that COVID-19 patients have a difficult time dealing with the disease and getting adapted to their new situation, leading to a remarkable decrease in the quality of their lives. Analyzing the transcribed interviews led to emergence of four main themes: "disbelief in being really ill," "fear and stress," "changes in lifestyle," and "seriously adhering to health guidelines."

Conclusion: Our results showed that COVID-19 patients suffer from physical pain and undergo poor mental health due to fear and stress resulting in worsening quality of life. A deep understanding of COVID-19 patients' states and situations is highly significant to help nurses and nursing managers plan effective strategies for caring for patients with COVID19.

Keywords: COVID-19 pandemic, phenomenological study, COVID-19 patients' lived experience

Received: //20

Accepted: 18/2/2021

Published: 30/05/2021

\section{INTRODUCTION}

COVID-19 emerged in 2019 and became an invisible assassin in 2020 which spread around the world. Since then, it has been threatening people's health and lives. It has also led to substantial economic and financial difficulties around the world [1]. The statistics of July 21, 2020, released by the World Health Organization. (WHO), revealed that there were $14,883,578$ positive cases of individuals with COVID-19, of which 8,937,163 recovered and 614,019 died. The same report showed that there were 94,693 positive cases of COVID-19 in Iraq by the same date, of which 62,836 recovered and 3,869 died. The report also showed 7,936 positive cases of COVID-19 in the Kurdistan region of Iraq, of which 3,233 recovered and 266 died [2].A very high rate of infection is one of the consequences of COVID-19, resulting in a high 
level of fear and anxiety of getting infected As a result, this pandemic caused almost all countries' lockdown and imposed strict restrictions on people's free movements [3]. It is necessary to focus and observe the emotions involved in a pandemic, such as anger and fear, to understand the psychiatric and psychological repercussions. As an adaptive animal defense mechanism and a key to survival, fear includes several biological processes that involve in readiness to respond to events that are potentially threatening. However, in case of disproportionate or chronic fear, it will become a harmful element that causes various psychiatric disorders to develop [4]. In a pandemic, stress and anxiety levels in healthy individuals rise due to fear, and preexisting psychiatric symptoms intensify [5]. The level of infection risk should be considered and protective steps need to be taken into account by individuals who are at higher risk of COVID-19 and those who live with them. In places where social distancing is challenging to maintain, and protective measures are impracticable, general rules need to be considered [6]. During epidemics, the number of those whose mental health is negatively affected is more than those affected by the infection; therefore, quality of life decreases dramatically among all people. As revealed by past tragedies, the mental health implications are more prevalent and can last longer than the epidemic itself. Moreover, the economic and psychosocial effects cannot be calculated if their resonance is considered in different contexts. In addition, mental disorders can lead to high economic costs. Appropriate strategies adopted to improve mental health may benefit the physical health and economic sectors [5].Since COVID-19 is a relatively new virus, there is limited understanding of the risk of being affected. In this regard, a study conducted in the Kurdistan region of Iraq revealed that protective behavior measures were adhered to the respondents reasonably. However, there was a relatively low frequency of undertaking some significant protective behaviors. It was also shown that protective behaviors were provoked by the risk perception of COVID-19 to a limited extent, such that it is a challenge to modify behaviors even for those who are well aware and well-educated [7].In addition to death and fear, the COVID-19 pandemic has negative impacts on families, schools, companies, public places, and work routines resulting in isolation and feelings of helplessness and abandonment. Moreover, its negative effects on the economy can raise the level of insecurity in society [8]. Many laboratory and histopathological tests have been performed to devise protocols to deal with the highly infective virus of COVID-19. These tests have largely neglected real-life experiences of COVID-19 patients' which are highly significant in developing strategies to improve patients' quality of life. [9].The investigation into the psychological issues and lived experiences of COVID-19 patients during their hospitalization has a high decisive impact on the process of recovery for these patients. Given the high prevalence of COVID-19 infection in the Kurdistan region of Iraq, this study was conducted to evaluate the lived experiences of COVID-19 patients during their hospitalization period to devise techniques to improve the quality of their lives.

\section{METHODS AND PATIENTS}

Study design: The present qualitative study was carried out by Van Manen's (1990) hermeneutic phenomenological method [10].Patients and setting: The study sample consisted of 12 COVID-19 patients hospitalized in Erbil's Emirates Hospital, the Kurdistan Region of Iraq. The study was conducted over three months from August 
2020 to September 2020. Patients were selected using a purposeful sampling method. The inclusion criteria were being diagnosed with COVID-19, age of over 18 years, willingness and consent to participate in the study, and having undergone treatment and hospitalization procedures. Exclusion criteria were lack of willingness or consent to participate in the study and inability to describe their lived experiences. Data collection: In-depth semi-structured interviews were conducted with the patients in order to collect the required data. For this purpose, a small relaxing room at Erbil's Emirates Hospital was used. The interviewers established a friendly relationship with the patients to elicit as much information as possible. Patients were informed and reassured about the confidentiality of their information. The duration of the interviews was flexible, ranging from 55 to 70 minutes, and enough time was given to the patients to answer the questions with as many details. To ensure patients' comfortability, they were interviewed by interviewers of the same gender. Predetermined questions used to direct the interviews included general questions such as "How is it like to suffer from COVID-19?", "When did you notice you had coronavirus?", "How did it feel in the beginning?", and "What is it like to have COVID-19?" and were followed by more specific questions such as "How has COVID19 changed your life?" or "What have been the effects of COVID-19 on your daily life?". With the patients' permission, all interviews were recorded and transcribed verbatim and then translated for further analysis. Data analysis: The transcripts of the interviews were analyzed through content a analysis approach. The accuracy of the translated transcripts was checked by comparing them to the recorded interviews several times. Afterward, holistic, detailed, selective approaches were used to break down the interviews into words, phrases, and sentences to depict the patients' lived experiences. Transcripts were reread and scrutinized several times to confirm understanding of patients' lived-experiences. Trustworthiness: Trustworthiness in qualitative studies refers to the level of soundness or adequacy [10]. To ensure a qualitative study's trustworthiness, it is essential to justify the results' reliability and describe the data analysis procedure [11]. In the current study, trustworthiness and the accuracy and rigor of the findings were ensured through peer checking conducted by two professors who also revised the transcripts and the extracted codes. Additionally, a friendly relationship between the participants and the researcher ensured the rigor of this study. Following data analysis, member checks, discussions of the study results between patients and the researcher, were conducted to obtain patients' feedback. Every stage of the study was monitored by two more expert professors in the field, and their suggestions were taken into account. Data collection was conducted during a period of 3 months to ensure prolonged engagement. Ethical considerations: Approval from the Ethics Committee of College of Nursing, Hawler Medical University (No. 7 on 26-72020) was obtained for this study. Patients were provided with sufficient information about the study's objectives, data collection methods, and confidentiality of the collected information. Patients were assigned a unique code to keep the collected data anonymous and to protect their confidentiality. All files were stored in a safe and secure place. Patients were informed of the right to abandon the study whenever they wanted to. Informed written consent were obtained from patients prior to the start of the study. 


\section{RESULTS}

Table 1 describes the patients' sociodemographic characteristics. The majority of the patients were males $(66.66 \%)$, over 36 years old $(75 \%)$, Muslims $(75.5 \%)$ and reside in urban areas (83.33\%). Of the patients, $33.33 \%$ had university degrees, $25 \%$ had high school degrees, $16.66 \%$ had primary degrees, and $25 \%$ were illiterate. Half of the patients had a good socioeconomic status (See Table 1).

Table 1: The patients' socio demographic data

\begin{tabular}{|c|c|c|}
\hline \multicolumn{2}{|c|}{$\begin{array}{c}\text { Socio-demographic and clinical } \\
\text { variables }\end{array}$} & \multirow{2}{*}{$\begin{array}{c}n(\%) \\
4(33.33)\end{array}$} \\
\hline \multirow{3}{*}{ Sex } & Female & \\
\hline & Male & $8(66.66)$ \\
\hline & $18-25$ & $1(8.33)$ \\
\hline \multirow{3}{*}{ Age (years) } & $26-35$ & $2(16.66)$ \\
\hline & $36-45$ & $4(33.33)$ \\
\hline & $46 \leq$ & $5(41.66)$ \\
\hline \multirow{4}{*}{ Place of residence } & Urban & $10(83.33)$ \\
\hline & Rural & $2(16.66)$ \\
\hline & Illiterate & $3(25.0)$ \\
\hline & Primary & $2(16.66)$ \\
\hline \multirow[t]{2}{*}{ Education level } & High school & $3(25.0)$ \\
\hline & University & $4(33.33)$ \\
\hline \multirow{2}{*}{ Religion } & Muslim & $9(75.5)$ \\
\hline & Christian & $3(25.0)$ \\
\hline \multirow{2}{*}{ Employment } & Employed & $8(66.66)$ \\
\hline & Unemployed & $4(33.33)$ \\
\hline \multirow{3}{*}{$\begin{array}{l}\text { Socioeconomic } \\
\text { status }\end{array}$} & Poor & $3(25.0)$ \\
\hline & Good & $6(50.0)$ \\
\hline & Very good & $3(25.0)$ \\
\hline
\end{tabular}

Content analysis of the scripts based on Van Manen's (1990) hermeneutic phenomenological method led to the emergence of four main themes: "disbelief in being really ill," "fear and stress," "changes in lifestyle," and "seriously adhering to health guidelines." These themes are presented in two major categories: before getting the disease and after getting the disease. Theme 1: Disbelief in being really ill Interviews with the COVID-19 patients revealed that some of them had not taken the disease seriously before they got it. Patients described their experiences in this regard as follows. "Before I got corona, I thought that this disease did not even exist, and it was just a political game played by the governments to control people, cut their salaries or wages, and even kill senior citizens to cut their pension." (Patient 3) "Before I developed the disease, I never wore a face mask or gloves because I did not take the disease seriously, and I believed that it did not exist and it was a rumor spread by governments all over the world. I really regret my wrong belief which caused me to be hospitalized here for more than 10 days." (Patient 7)

"I didn't trust the media and statistics of coronavirus until I myself got the disease. In the beginning, I couldn't believe, so I visited 2 different clinics, and finally came here. They all confirmed that I had corona disease." (Patient 9)

Theme 2: Fear and stress

Patients' accounts of their experiences revealed that COVID-19 caused immense fear and stress in their lives. Their fear and stress were related to the probability death and concerns of the disease spreading among their family members. Patients described their experiences in this regard as follows. "Once I was diagnosed with coronavirus, I was filled with fear and stress. I was really scared to die. I had a lot of stress to transfer the disease to my 
family members. It's a really painful disease, and during my treatment, I felt I was going to die many times."(Patient 1).

"It's a really painful and stressful disease. Its pain is not just physical, it is also mental. The physical side is related to the pain it causes in your muscles, legs, chest, and eyes. Its mental pain is related to the fear and stress it causes because of your family members and the society which consider coronavirus as a shame and almost everybody gets away from you, even your dearest ones."(Patient 10). "Before I got the disease, I heard and read a lot about its pain, and when I was told that my coronavirus test was positive, I got quite shocked and felt fear and stress deep inside. I feared that I might transfer the disease to my family members. I was also filled with a feeling of shame because our society has really negative attitude toward this disease." (Patient 12)Theme 3: Changes in lifestylePatients stated that COVID-19 had changed their lives significantly, and they had to live in a quite different way. In their accounts, they talked about changes in their sleep, food, physical activities, and special treatment. Patients described their experiences in this regard as follows.

"Being a corona patient means to live a quite new life. I used to sleep about 5 to 6 hours, but since I got COVID-19, I have to sleep about 10 hours, following my doctor's order. I'm a quite sociable person, and I like to spend most of my time with my family members and friends, but because of my disease I haven't seen anyone in person for more than two weeks. I only speak to them on the phone."(Patient 2)

"I didn't use to do any exercise before I got corona, but these days I'm doing exercise for about half an hour every day. It's a part of my treatment. I've also had to change my eating habits. Nowadays I'm eating a lot of watery food and fruits like watermelon and cucumber. I also drink a lot of water." (Patient 5). "The treatment has been a long procedure. I remember on the first days of my hospitalization, I couldn't breathe without the ventilator. It's really hard not to be able to breathe in or out. I'm an active person, but I've been here on this bed for more than 8 days without any activities, which is really boring." (Patient 8)Theme 4: Seriously adhering to health guidelines The final theme that emerged from the patients' stories was their emphasis on following the guidelines recommended by the Ministry of Health. Patients described their experiences in this regard as follows." I remember I always made jokes about those who followed the health guidelines and were wearing face masks and gloves until I myself got the disease and I came to know that this disease is quite serious and fatal, so I recommend all my friends and people to follow the health guidelines." (Patient 4)

"If I had followed the health guidelines recommended by the Ministry of Health, I wouldn't have got the disease. So, I recommend everybody that they take corona seriously, it's really debilitating, and its treatment is quite costly." (Patient 5)

"If you love your life, family, and friends, please adhere the health guidelines, wear face masks and gloves, wash your hands regularly, eat healthy food, drink a lot of water, and more importantly keep social distance. I wish I had adhered all the guidelines recommended by the Ministry of Health." (Patient 11)

\section{DISCUSSION}

The spread of COVID-19 is remarkably high, and it results in uncommon effects. The symptoms of COVID-19 include shortness of breath, dry cough, aches, and fever. It can also lead to respiratory failure and dysfunction of different organs [12]. Its adverse effects have been observed on all sectors of society, including education, 
economy, and food. Successful dealing with the COVID-19 pandemic and its impacts on society requires collective efforts and cooperation of organizations, governments, and individuals [13]. Adverse effects of COVID-19 on physical and mental health continues to threaten quality of life [14]. Supporting COVID-19 patients with their lifestyle can improve the quality of their lives. In this study, nearly all patients stated that prior to being infected with COVID19 they did not believe that COVID-19 existed. Rather, they believed it was a political game by the government used to control people. This finding aligns with Uscinski et al. (2020) which reported people's belief in COVID-19 conspiracy theories. Uscinski et al. found that believing in such theories were rooted in psychological predispositions resulting in distrust of information distributed by experts and other authorities as well as believing that major events like the COVID-19 pandemic are products of conspiracies, and ideological and partisan motivations. Uscinski et al. further remarked that such conspiracy beliefs have negative effects on devising and adhering to treatment as well as developing coping strategies to reduce the consequences of such events [15]. As the results of the present study indicated, considering COVID-19 as a political game or a conspiracy led to individuals being infected by the virus. Almost all countries in the world are currently affected by the COVID-19 pandemic. The number of COVID-19 patients in the Kurdistan region of Iraq continues to increase. The second theme in the present study was fear and stress felt by both patients and heathy individuals. In this regard, it is stated that since COVID-19 is highly infectious, people in affected countries are suffering from fear, stress, and anxiety which in turn threaten their mental health and wellbeing [14], and the negative attitude of the community toward COVID-19 disease and patients worsen these critical situations [16]. To prevent infection of more people who might surge to hospitals, home treatment and quarantine are the best solutions for reliable and educable patients who have mild or no symptoms and observe all due diligence. Quarantining in the home which is one of most effective control measures for epidemic diseases can also reduce people's stress and fear due to being with family members and being helped by them $[17,18]$.It was also stated that the patients felt shameful to have COVID-19 disease. In this regard, it is stated that quarantine and restricted interpersonal relationships may result in senses of shame and loneliness which can be tackled by taking these measures for the purpose of recovery and family reunion. In addition, psychiatric nurses are recommended to develop programs in the form of psychological support or comprehensive spiritual care packages and utilize multiple media channels to help patients overcome such feelings [19].The third theme was found to be changes in lifestyle of patients and people. This finding is also emphasized by other studies which showed that the COVID19 pandemic has disrupted trades and movements all over the world and negatively impacts day-to-day businesses and life. Dramatic changes can happen in dietary and sleep patterns as a result of adhering to quarantine rules and can cause additional stressful conditions or exacerbate existing stressful conditions[20,21]. Such stress can be the result of people's worry about their next paycheck, shortage of food on the grocery shelves, and staying at home all day long [22]. Additionally, both physical and mental health can negatively be influenced by poor quality sleep. The ability of the immune system to resist infections can also decrease. In this regard, it is recommended that electronic devices should be turned off, watching news needs 
to be minimized, alcoholic and caffeine drinks should be avoided, and regular schedule for sleeping needs to be made [23]. The patients in the current study referred to complete changes in their lifestyles due to COVID-19 pandemic. In a similar study, it is mentioned that people's day-to-day life around the world has changed due to destructive effects of COVID-19 pandemic. It is also stated that taking up a new lifestyle and being provided with special treatment are keys to improvement in the quality of their lives. Moreover, adhering to health guidelines such as wearing masks and gloves, washing hands regularly, eating and drinking healthy, and keeping social distance are key to preventing COVID-19 [24]. In addition, remote conduction of business training and assessments needs to be taken into account to avoid gatherings $[25,26]$.It has been stated that social media can provide the public with disseminating information, and many individuals will experience isolation during hospitalization or quarantine at home $[27,28]$. However, social media needs to turn into an effective source of information and means for keeping abreast of the broad amount of medical knowledge [29]. Countries with humanitarian-cooperative culture, low education levels, and pronounced social disparity are reported to have no parameters for estimating the effects of COVID19 on the population's behavior or mental health .8 As a result, it is necessary to provide health guidelines aimed at improving individuals' mental health in such countries [30].The present study had some limitations. The first limitation is related to the limited number of the patients who participated in the present study, which limits the generalizability of the findings to other groups of people in other places. However, due to the situation of COVID-19 patients, it was almost impossible to recruit more patients. Another limitation is related to the relatively short duration of engagement with the patients and data collection, which can affect the reliability and validity of the findings because trust and good relationship which form over time and are vital for phenomenological studies can highly be affected.

\section{CONCLUSION}

COVID-19 patients have a poor quality of life which is even worsened as a result of fear and stress. A deep understanding of such patients' situations is vital to help nurses and nursing managers plan effective strategies to provide care to patients with COVID-19. This includes making changes in their lifestyle, encouraging them to adhere to health guidelines, and providing them with psychological support and appropriate treatment.

\section{Competing interests}

The author declares no competing interests.

\section{REFERENCES}

[1] Byrne L, Wykes T. A role for lived experience mental health leadership in the age of Covid19. Journal of mental health. 2020;29(3):243 -6.https:// doi.org/10.1080/09638237.2020.1766002

[2] Coronavirus Disease (COVID-19) Situation Reports [Internet]. Who.int. [cited 2020 Aug 25]. Available from: https://www.who.int/ emergencies/diseases/novel-coronavirus2019/situation-reports .

[3] Cascella M, Rajnik M, Cuomo A, Dulebohn SC, Di Napoli R. Features, Evaluation, and Treatment of Coronavirus. 2020 Oct 4. In: StatPearls [Internet]. Treasure Island (FL): StatPearls Publishing; 2020 Jan-. PMID: 32150360.

[4] Garcia R. Neurobiology of fear and specific phobias. Learning and memory. 2017;24 (9):462-71.

[5] Shigemura J, Ursano RJ, Morganstein JC, Kurosawa M, Benedek DM. Public responses to the novel 2019 coronavirus (2019-nCoV) in Japan: mental health consequences and 
target populations. Psychiatry and clinical neurosciences; 2020;74:281-2.

[6] Sahoo S, Mehra A, Suri V, Malhotra P, Yaddanapudi LN, Dutt Puri G, et al. Lived experiences of the corona survivors (patients admitted in COVID wards): A narrative real -life documented summaries of internalized guilt, shame, stigma, anger. Asian journal of psychiatry. 2020;53 (102187):102187.

[7] Shabu S, Amen KM, Mahmood KI, Shabila NP. Risk perception and behavioral response to COVID-19 in Iraqi Kurdistan Region [Internet]. Research square. 2020. http://dx.doi.org/10.21203/rs.3.rs-22025/ v1.

[8] Ornell F, Schuch JB, Sordi AO, Kessler FHP. "Pandemic fear" and COVID-19: mental health burden and strategies. Brazilian journal of psychiatry. 2020;42(3):232-5. http://dx.doi.org/10.1590/1516-44462020-0008.

[9] Aziz PY, Hadi JM, Sha AM, Aziz SB, Rahman $\mathrm{HS}$, Ahmed HA, et al. The strategy for controlling COVID-19 in Kurdistan Regional Government (KRG)/Iraq: Identification, epidemiology, transmission, treatment, and recovery. International journal of surgery open. 2020;25:41-6.

[10] Holloway I, Wheeler S. Qualitative Research in Nursing and Healthcare. 2010. Oxford: Wiley-Blackwell.

[11] Elo S, Kyngäs $H$. The qualitative content analysis process. Journal of advanced nursing. 2008;62(1):107-15. http://10.1111/ j.1365-2648.2007.04569.x.

[12] Weible CM, Nohrstedt D, Cairney P, Carter DP, Crow DA, Durnová AP, et al. COVID-19 and the policy sciences: initial reactions and perspectives. Policy sciences. 2020;53 (2):1-17. https://doi.org/10.1007/s11077020-09381-4.

[13] Adolph C, Amano K, Bang-Jensen B, Fullman N, Wilkerson J. Pandemic politics: Timing state-level social distancing responses to COVID-19. Journal of health politics, policy and law Internet]. 2020; Available from: http:// dx.doi.org/10.1215/03616878-8802162.

[14] Liu, X., Kakade, M., Fuller, C.J., Fan, B., Fang, Y., Kong, J., et al. Depression after exposure to stressful events: lessons learned from the severe acute respiratory syndrome epidemic. Comprehensive psychiatry. 2012;53(1):15-23.
[15] Uscinski JE, Enders AM, Klofstad CM, Seelig M, Funchion J, Everett C, et al. Why Do People Believe COVID-19 Conspiracy Theories? The Harvard Kennedy School Misinformation Review [Internet]. 2020; Available from: http://dx.doi.org/10.37016/mr-2020-015.

[16] Duan, L., Zhu, G. Psychological interventions for people affected by the COVID-19 epidemic. The lancet psychiatry. 2020;7(4):300302. http://10.1016/S2215-0366(20)30073-0

[17] World Health Organization (WHO). Home care for patients with Middle East respiratory syndrome coronavirus (MERS-CoV) infection presenting with mild symptoms and management of contacts: interim guidance. World Health Organization; 2018: 2.3.2020.

[18] Li D. Challenges and responsibilities of family doctors in the new global coronavirus outbreak. Family medicine community health. 2020;8(1):e000333. https://10.1136/fmch2020-000333.

[19] Asgari P, Jackson AC, Bahramnezhad F. Resilient care of the patient with COVID-19 in Iran: A phenomenological Study [Internet]. Research square. 2020. Available from: http://dx.doi.org/10.21203/rs.3.rs-24733/ v1.

[20] Jin YH, Cai L, Cheng ZS, Cheng H, Deng T, Fan $Y P$, et al. A rapid advice guideline for the diagnosis and treatment of 2019 novel coronavirus (2019-nCoV) infected pneumonia (standard version). Military medical research. 2020;7(1), $4 . \quad$ https:// doi.org/10.1186/s40779-020-0233-6.

[21] Haleem, A., Javaid, M., Vaishy, R. and Vaishb, A. Effects of COVID-19 pandemic in the field of orthopaedics. Journal of clinical orthopaedics and trauma. 2020;11(3):498499. https://10.1016/j.jcot.2020.03.015.

[22] Brooks SK, Webster RK, Smith LE, Woodland L, Wessely S, Greenberg N, et al. The psychological impact of quarantine and how to reduce it: rapid review of the evidence. Lancet. 2020;395(10227):912-20. https:// doi.org/10.1016/S0140-6736(20)30460-8.

[23] Abdurahman AA, Bule M, Shab-Bidar S, Rezaei S, Djafarian K. The association between sleep duration and risk of abnormal lipid profile: a systematic review and metaanalysis. Obesity medicine. 2020;18:100236. https://doi.org/10.1016/ j.obmed.2020.100236.

[24] Haleem A, Javaid M. Effects of COVID-19 pandemic in daily life. Current medicine research and practice. 2020;10(2):78-9. 
doi: https://10.1016/j.cmrp.2020.03.011.

[25] Guidance for surgeons working during the COVID-19 pandemic [Internet]. Rcseng.ac.uk. [cited 2020 Aug 20]. Available from: https://www.rcseng.ac.uk/ coronavirus/joint-guidance-for-surgeonsv1.

[26] OSHA Guidance on Preparing Workplaces for COVID-19. 2020. U.S. Department of Labor Occupational Safety and Health Administration, OSHA 3990-03 2020.

[27] Ahmad AR, Murad HR. The Impact of Social Media on Panic During the COVID-19 Pandemic in Iraqi Kurdistan: Online Questionnaire Study. Journal of medical internet research. 2020;22(5): e19556). https://10.2196/19556.

[28] Pappot N, Taarnhøj GA, Pappot H. Telemedicine and e-Health Solutions for COVID -19: Patients' Perspective. Telemedicine journal and e-health. 2020;26(7):847-9. https://10.1089/tmj.2020.0099.

[29] McGowan BS, Wasko M, Vartabedian BS, Miller, RS, Freiherr, DD, Abdolrasulnia, M. Understanding the factors that influence the adoption and meaningful use of social media by physicians to share medical information. Journal of medical internet research. 2020;14(5):e117.doi: https://10.2196/jmir.2138.

[30] Dieltjens, T., Moonens, I., Van Praet, K., De Buck, E. and Vandekerckhove, P. A systematic literature searches on psychological first aid: lack of evidence to develop guidelines. Plos one. 2014;9(12), e114714. https://doi.org/10.1371/ journal.pone.0114714. 